\title{
Puberty menorrhagia in modern era: analysis in a tertiary care centre
}

\author{
Prachi Saurabh Koranne*, Aparna R. Wahane
}

Department of Obstetrics \& Gynaecology, Government Medical College, Akola-444005, Maharashtra, India

Received: 28 June 2014

Accepted: 5 July 2014

\section{*Correspondence:}

Dr. Prachi Saurabh Koranne,

E-mail: prachibs81@gmail.com, prachi_koranne@yahoo.com

(C) 2014 Koranne PS et al. This is an open-access article distributed under the terms of the Creative Commons Attribution Non-Commercial License, which permits unrestricted non-commercial use, distribution, and reproduction in any medium, provided the original work is properly cited.

\begin{abstract}
Background: Puberty menorrhagia can pose a significant challenge to the gynecologist when associated with serious systemic complications like anaemia and hypoproteinaemia. Early diagnosis and treatment with individualization of each and every case is the keystone in the management of puberty menorrhagia. Exclusion of pregnancy is mandatory in every case, irrespective of the history, reassurance, counseling, regular follow-up along with a balanced nutritional diet and long term iron therapy go a long way in successful management of such cases.

Methods: Data was collected from medical case records in each of these cases from indoor case sheets.

Results: There were 35 indoor admissions in the gynaecology ward at GMC Akola 2011 to 2013 for critical puberty menorrhagia over a span of two years. The leading cause was anovulatory dysfunctional uterine bleeding. Other systemic associations included hypothyroidism, idiopathic thrombocytopenic purpura, genital tuberculosis, and PCOD. Each case was analyzed for demographic profile, duration of menorrhagia, severity of symptoms, degree of anaemia, final diagnosis, requirement of blood and component therapy and response to conservative management.

Conclusions: Most abnormal bleeding in adolescents is caused by immaturity of the hypothalamic - pituitary ovarian axis resulting in anovulation. Approximately $20 \%$ of adolescents have an underlying endocrine or haematological disorder requiring targeted diagnostic testing.
\end{abstract}

Keywords: Puberty menorrhagia, Bleeding disorders, Dysfunctional uterine bleeding, Hormonal therapy

\section{INTRODUCTION}

Menarche is a hallmark event in the life of most adolescent girls. It marks the transition from childhood to puberty. Although mechanisms triggering puberty and menarche remain uncertain, they are dependent on genetics, nutrition, body weight and maturation of the hypothalamic pituitary- ovarian axis. The complete maturation of the axis may take up to 2 years. During this time, it is common for adolescents to present with complaints of menstrual irregularities.

Abnormal bleeding accounts for approximately 50\% of gynaecological visits in adolescent girls. ${ }^{1}$ These complaints encompass disorders ranging from minimal spotting to profuse bleeding. Puberty menorrhagia is defined as excessive bleeding occurring between menarche and 19 years. In $80 \%$ of cases puberty menorrhagia is caused by anovulatory cycles. ${ }^{3}$

There is an immaturity of the hypothalamus and inadequate positive feedback resulting in sustained high levels of estrogen. An organic disease or malignancy in particular is very rare.

In all cases of puberty menorrhagia it is mandatory to exclude pregnancy, especially an incomplete abortion or ectopic pregnancy. In persistent abnormal bleeding coagulation disorders and leukemia should be ruled out. Occasionally menorrhagia is the only presenting symptom in a patient of coagulation disorders. ${ }^{2}$ In general the prognosis is better when dysfunctional uterine 
bleeding starts after a period of regular menstruation than when it starts at menarche. ${ }^{4}$

\section{METHODS}

The present ongoing study evaluates 35 cases of critical puberty menorrhagia managed by domiciliary care at Government medical college Akola, over a span of 2 years from January 2011 to December 2013. Data was collected from medical case records in each of these cases from indoor case sheets. A proforma was made and data entered and analyzed from each proforma. An approval was taken from ethical committee of hospital. It was a retrospective analytical study and no intervention was done.

Each case was evaluated for the demographic profile, severity of symptoms, degree of anaemia, final diagnosis, requirement of blood and component therapy and response to conservative management. The baseline investigations in all the cases included exclusion of pregnancy by urine testing, complete blood count, peripheral smear for RBC and WBC morphology, coagulation profile, blood grouping and $\mathrm{Rh}$ typing and transabdominal USG. In selected cases thyroid function test $\left(\mathrm{T}_{3}, \mathrm{~T}_{4}, \mathrm{TSH}\right)$ and hormonal assays including $(\mathrm{LH}$, FSH, Prolactin) and chest X-ray were done.

\section{RESULTS}

Analysis of data (Table 1) showed that $50 \%$ of patients were in the age group of 13-15 years.

Table 1: Analysis of data.

\begin{tabular}{|ll|}
\hline $\begin{array}{l}\text { Age group } \\
\text { (years) }\end{array}$ & Percentage \\
\hline $13-15$ & $50 \%$ \\
\hline $15-17$ & $25 \%$ \\
\hline $17-19$ & $25 \%$ \\
\hline
\end{tabular}

\section{Socioeconomic status}

Majority of the patients belonged to the lower socioeconomic status.

As shown in Table 2 almost $62 \%$ of patients had onset of menorrhagia since less than 6 months. Of these 33\% of patients had haemoglobin less than $6 \mathrm{Gm} \%$.

\section{Table 2: Socioeconomic status.}

\begin{tabular}{|ll|}
\hline Duration & Percentage \\
\hline Less than 6 months & $62 \%$ \\
\hline 6 months to one year & $21 \%$ \\
\hline More than 1 year & $17 \%$ \\
\hline
\end{tabular}

As shown in Table 3 anovulatory dysfunctional uterine bleeding occurred in 27 cases $(80 \%)$. Two patients of secondary DUB had a final diagnosis of idiopathic thrombocytopenic purpura, 2 patients were diagnosed to have hypothyroidism. Genital tuberculosis was detected in another 2 cases. One patient was found to have a rare cause - Glanzmanns thromboasthenia that is discussed later.

Table 3: Causes of puberty menorrhagia.

\begin{tabular}{|lll|}
\hline Causes & $\begin{array}{l}\text { No. of } \\
\text { cases }\end{array}$ & $\%$ \\
\hline Anovulatory dysfunctional uterine bleeding & 27 & $80 \%$ \\
\hline Idiopathic thrombocytopenic purpura & 2 & $5.7 \%$ \\
\hline Hypothyroidism & 2 & $5.7 \%$ \\
\hline Genital tuberculosis & 1 & $2.8 \%$ \\
\hline PCOD & 2 & $5.7 \%$ \\
\hline Glazmann thrombosthenia & 1 & $2.8 \%$ \\
\hline
\end{tabular}

Analysis of data showed that $37 \%$ of patients received fresh blood transfusion, $8 \%$ required component therapy in the form of fresh frozen plasma, platelets and cryoprecipitates. Majority of patients received a combination medical therapy as shown in the following table.

\section{Combination medical therapy regimes}

Majority of patients received OC pills + ethamsylate (20\%). Six patients received i.v. oestrogens plus progesterone while ethamsylate along with progestogens was used in 5 patients. Tranexamic acid was used with biphasic OC pills in $17.1 \%$ of cases and with progestogens in $8.5 \%$ cases.

Table 4: Combination medical therapy regimes.

\begin{tabular}{|lll|} 
Therapy regimen & $\begin{array}{l}\text { No. of } \\
\text { patients }\end{array}$ & $\%$ \\
\hline Biphasic OC pills + ethamsylate & 7 & 20 \\
\hline Biphasic OC pills + tranexamic acid & 6 & 17.1 \\
\hline IV estrogen + progestogen & 6 & 17.1 \\
\hline OC pills + testosterone in acute phase & 6 & 17.1 \\
\hline Progestogen + ethamsylate & 5 & 14.2 \\
\hline Progestogen + tranexamic acid & 3 & 8.5 \\
\hline Progestogen & 2 & 5.7 \\
\hline
\end{tabular}

\section{DISCUSSION}

Puberty menorrhagia is excessive bleeding occurring between menarche and 19 years of age. It severely affects the quality of life. ${ }^{5}$

The main causes of abnormal uterine bleeding adolescent are $^{6}$

\section{Uterine causes}

Pregnancy, endometritis, hyperplasia, malignancy, polyp, leiomyomata 


\section{Ovarian causes}

Immature hypothalamic pituitary ovarian axis, pcod, estrogen producing tumors

\section{Cervical causes}

Cervicitis, condyloma, sarcoma botryoides, polyp, malignancy

\section{Vulval/vaginal causes}

Trauma, vaginitis, infection, sarcoma botryoides

\section{Endocrine causes}

Hypothyroidism, hyperprolactinaemia

\section{Coagulation disorders}

In the present study series in $80 \%$ of cases of puberty menorrhagia the cause was found to be anovulatory dysfunctional uterine bleeding. A review of literature shows that during puberty, maturation of the hypothalamic pituitary - ovarian axis is characterized by an increase in the frequency and amplitude of pulsatile $\mathrm{GnRH}$, which initiates and regulates secretion of pituitary gonadotropins. ${ }^{9}$

During the prepubertal years, LH is secreted primarily at night in an episodic fashion. With the progression to puberty, LH peaks increase in a pattern similar to that seen at night. The timing of these LH pulses is crucial in establishing normal ovulatory cycles. Increases in basal LH as well as immature timing of pulses result in anovulatory cycles. These cycles are characterized by levels of LH and FSH secretion that are sufficient to induce follicular development and estrogen production but inadequate to induce follicular maturation and ovulation. Thus unopposed estrogen stimulates endometrial growth. This ultimately outgrows its blood supply and architectural support, resulting in partial breakdown and shedding in an irregular manner.

In the proliferative phase the endometrium synthesizes equal amounts of $\mathrm{PGE}_{2 \alpha}$ (Vasoconstrictor and weak platelet aggregator) and $\mathrm{PGE}_{2}$ (Vasodilator with weak platelet antiaggregatory effect). However in the luteal phase the levels of $\mathrm{PGE}_{2 \alpha}$ progressively increase under the influence of estradiol and progesterone. In normal menstruation the ratio of $\mathrm{PGE}_{2 \alpha}: \mathrm{PGE}_{2}$ is $2: 1$ so that it is the vasoconstrictor and platelet aggregator action that predominates. In anovulatory DUB the lack of progesterone results in decrease in the $\mathrm{PGE}_{2 \alpha}: \mathrm{PGE}_{2}$ ratio and relative increase in the vasodilator and antiplatelet aggregatory $\mathrm{PGE}_{2}$ which would account for the increased mean menstrual blood loss. It could also account for the absence of uterine contractions and painless periods characteristics of anovulatory menstruation.

In the present series of patients, majority were administered a combination medical regime, to control the acute phase of bleeding. The primary management of anovulatory bleeding should be directed at controlling symptoms and prevention of anaemia. In adolescents with mild bleeding, reassurance and prophylactic iron treatment are suitable. However in patients who are sexually active or symptomatic for 3 to 6 months with anaemia (Hb 9-12 $\mathrm{mg} / \mathrm{dl})$ require cyclical medroxy progesterone acetate or a monophasic oral contraceptive pill in addition to iron. If the patient is actively bleeding, but haemodynamically stable, the acute phase is managed with one oral contraceptive pill three times daily for 3 days followed by two oral contraceptive pills twice daily for 2 days; this is followed by one oral contraceptive pills until completion of the packet. The cycles are further stabilized by a course of oral contraceptive for 3 to 6 months. If the patient continues to bleed heavily after the first 3 days, treatment is extended to three times daily for 7 days, twice daily for 7 day and then daily as mentioned previously. In patient with severe bleeding associated with hemodynamic changes, blood transfusions are indicated with administration of intravenous conjugated oestrogens (e.g. premarin) in a dose of $25 \mathrm{mg}$ every 4 to 6 hours until bleeding stops for 24 hours. ${ }^{13}$ Once bleeding is controlled, the patient is started on a regimen of strong androgenic progestogen or oral contraceptive pills.

The occurrence of excessively heavy irregular menses should prompt an evaluation of haematological status to rule out blood dyscrasias. In the present study group 3 patients $(8.5 \%)$ had blood dyscrasias manifesting as DUB. Claessens and Cowell reported 19\% of adolescents with menorrhagia requiring hospitalization had an underlying coagulation disorder in their study. ${ }^{6}$ A more recent retrospective study by Falcone et al in 1994 found that $4.9 \%$ of admissions over a 10 year period were secondary to a coagulopathy. ${ }^{9}$

The most common coagulation disorders were idiopathic thrombocytopenic purpura, Von Willebrands disease, and leukemia and platelet dysfunction like Glanzmanns thromboasthenia. Young girls with blood coagulopathies are at a high risk for abnormal bleeding with the onset of menarche, ${ }^{9}$ and must be treated appropriately at the time of puberty. Laboratory evaluation, including a complete blood count, platelets, prothrombin time, partial thromboplastin time and bleeding time provides an adequate screen for coagulation disorders.

In our study there were 2 patients diagnosed to have idiopathic thrombocytopenic purpura. $80 \%$ patients of idiopathic thrombocytopenic purpura have menorrhagia. ${ }^{4}$ Acute Idiopathic thrombocytopenic purpura is most commonly seen in young and is immunological thrombocytopenia, caused by immuno complexes containing viral antigens that bind to the platelet, Fc receptors, or, by antibodies produced against viral antigens that cross react with platelets. It can be associated with infectious mononucleosis, acute toxoplasmosis, CMV infections, viral hepatitis and HIV. ${ }^{11}$ 
In the present study group there was one rare case of Glanzmanns thromboasthenia. This is a disorder in which the IIb-IIIa complex glycoprotein on the platelet surface is defective and platelets cannot form aggregates. Our case required multiple platelet transfusions. All haematological disorders underlying dysfunctional uterine bleeding cases are best treated in liaison with the haematologist. They require adequate blood transfusion, component therapy and platelet transfusion.

Hypothyroidism can be associated with pubertal DUB. The reported incidence of subjective menorrhagia in myxoedema varies from $32-80 \%$ and menorrhagia may not infrequently be the presenting complaint (Scoot and Massey 1964). The menorrhagia associated with hypothyroidism responds promptly to the thyroid replacements, often in doses insufficient to correct the other manifestations of the condition. This suggests that thyroxine does have a direct effect on the spiral arterioles and on haemostasis at menstruation. ${ }^{4}$

In our study there were two cases of genital TB which were treated with anti-Kochs therapy along with OCPs. Of these one case presented initially with idiopathic thrombocytopenic purpura and was detected to have genital tuberculosis on further evaluation. The incidence of genital TB is about $1 \%$ amongst the gynaecological patients attending the ODP in developing countries. ${ }^{10}$ Menorrhagia or irregular bleeding in genital TB is probably due to ovarian involvement, pelvic congestion or endometrial lesions.

Polycystic ovarian disease can be infrequently associated with irregular heavy bleeding in $30 \%$ of cases, as reported by Goldzeiher et al. Chronic anovulation may result from an increased pulsatility of GnRH. This results in elevated LH levels and increased ovarian androgen production. These hormone increases result in menstrual irregularity and oily skin. Ovaries typically have multiple follicular cysts less than $10 \mathrm{~mm}$ in size and increased stroma. This condition may be temporary in adolescent or may eventually progress to advanced polycystic ovarian disease with hirsuitism. Peripheral conversion of androstenedione to oestrone causes a hyperoestrogenic effect that perpetuates menstrual irregularity.

The goals of treatment in adolescents are to regulate menstruation and decrease hirsuitism and acne. The best treatment modality is an oral contraceptive pill because of the inhibition of $\mathrm{LH}$ and decrease in circulating testosterone levels. Sex hormone -binding globulin is increased and available to bind and inactivate testosterone in the circulation. ${ }^{7}$ Oral contraceptive pills do not seem to aggravate the underlying insulin resistance significantly and may attenuate some of the lipid derangement's induced by sustained excess androgen exposure $^{8}$ Another treatment option to control abnormal bleeding is cyclic medroxyprogesterone acetate $10 \mathrm{mg}$ orally for 10 days of each month. However this regimen does not alleviate the associated androgenic effects of polycystic ovaries. ${ }^{9}$
To summarize, Blood and component therapy along with hormones constitute the main medical therapy in the treatment of critical puberty menorrhagia. In the present study 6 patients required intravenous conjugated estrogen. Various studies suggest that these are of value in arresting profuse haemorrhage 4 and are usually given with antiemetics. Oral contraceptive pills were administered to 19 patients in our study group. These form an effective hormonal therapy to restore the balance between prostaglandins and thromboxane $\mathrm{A}_{2}$ and reduce the mean menstrual blood loss with additional cycle stabilization. $45.7 \%$ patients in our study series had received progestogens for medical curettage. Androgenic progestogens alone may be used to arrest uterine haemorrhage or administered cyclically throughout the menstrual cycle $\left(5^{\text {th }}\right.$ to $25^{\text {th }}$ day) norethisterone acetate (primolut $\mathrm{N}$ ) $20-30 \mathrm{mg}$ daily is given to arrest haemorrhage and not more than 3 days. The progestogen may then be continued at a lower dose for upto 21 days. The patient should be warned that a withdrawal bleeding will occur on stopping treatment that will cease in 4-5 days. Norethisterone acetate can also be given from $5^{\text {th }}$ to $25^{\text {th }}$ day in a dose of $5 \mathrm{mg}$ once a day.

Androgens like danazol are less favoured because of their masculinizing side effects in adolescent girls.

Antifibrinolytic like tranexamic acid are a newer form of treatment in puberty menorrhagia. Plasminogen activators are a group of enzymes that cause fibrinolysis. An increase in the levels of plasminogen activators has been found in the endometrium of patients with heavy menstrual bleeding compared to those with normal menstrual loss. Plasminogen activators have been therefore been prompted as a treatment in heavy menstrual bleeding. ${ }^{3}$

There has been reluctance to prescribe tranexamic acid due to possible side effects of the drugs such as thrombogenic disease (DVT). Long term studies in Sweden; however have shown that the rate of incidence of thrombosis in women treated with tranexamic acid is comparable with the spontaneous frequency of thrombosis in women. ${ }^{3}$ Ethamsylate was used in 8 of our patients. It reduces capillary bleeding when the platelets are adquate; probably it corrects abnormal platelet function. It is not an antifibrinolytic. It does not stabilize fibrin.

Majority of patients in the study group received a combination therapy with OCPs with androgens or with progesterone or with antifibrinolytic agents. ${ }^{12}$

\section{CONCLUSION}

In conclusion, most abnormal bleeding in adolescents is caused by immaturity of the hypothalamic - pituitary ovarian axis resulting in anovulation. Approximately $20 \%$ of adolescents have an underlying endocrine or 
haematological disorder requiring targeted diagnostic testing.

Individualizing every case, excluding pregnancy, timely hospitalization, a thorough history, physical examination and base line workup are crucial in the management of every case. Reassurance, counseling of adolescent girls about reproductive physiology, regular follow-up, balanced diet and long term iron therapy go a long way in treatment of puberty menorrhagia.

Funding: No funding sources Conflict of interest: None declared

Ethical approval: The study was approved by the hospital ethics committee

\section{REFERENCES}

1. Caufriez A. Menstrual disorders in adolescence: Pathophysiology and treatment. Horm Res. 1991;36:156.

2. Devore GR, Owens O, Kase N. Use of intravenous premarin in the treatment of dysfunctional uterine bleeding: a double blind randomized control study. Obstet Gynaecol. 1982;59:285.

3. Lethaby A, Farquhar C, Cooke I. Antifibrinolytics for heavy menstrual bleeding. Cochrane Database Syst Rev. 2000;(4):CD000249.

4. Davey DA. Dysfunctional Uterine Bleeding. In: C. R. Whitfield, eds. Dewhurst's Textbook of Obstetrics and Gynaecology for Postgraduates. 5th ed. New York: Wiley, John \& Sons; 1995: 591-607.

5. Royal College of Obstetricians and Gynaecologists. RCOG guideline for management of menorrhagia after referral to secondary care. In: RCOG, eds.
National Evidence Based Clinical Guideline No 5. London: RCOG; 1999: 1-134.

6. Claessens EA, Cowell CA. Acute adolescent menorrhagia, Am J Obstet Gynaecol 1981;139:277.

7. Raj SG, Raj MH, Talbert LM, Sloan CS, Hicks B. Normalization of testosterone levels using a low estrogen containing oral contraceptive in women with polycystic ovary syndrome. Obstet Gynaecol 1983;60:15.

8. Korytkowski MT, Mokan M, Horwitz MJ, Berga SL. Metabolic effects of oral contraceptives in women with polycystic ovary syndrome. J Clin Endocrinal Metab. 1996;80:517.

9. Debra A, Minjarez MD, Karen D, Bradshaw MD. Abnormal uterine bleeding in adolescents. Obstet Gynaecol Clin North Am. 2000;27(1):63-8.

10. Dutta DC. Pelvic infections: genital tuberculosis. In: Dutta DC, eds. Textbook of Gynaecology. 2nd ed. Kolkata, India: New central book agency (P) Ltd; 1990: 128-134.

11. Robert I. Handin. Disorders of the platelet and vessel wall. Harrisons Principles of Internal Medicine. In: Eugene Braunwald, Anthony S. Fauci, Dennis L. Kasper, Stephen L. Hauser, Richard M. Stone, Dan L. Longo, J. Larry Jameson, eds. 15th ed. New York: McGraw-Hill Companies; 2001: 745-750.

12. Tripathi KD. Drugs affecting coagulation, bleeding and thrombosis. In: Tripathi KD, eds. Essentials of Medical Pharmacology. 4th ed. New Delhi, India: Jaypee Brothers Medical Publishers; 1999: 599.

13. Duflos-Cohade C, Amandruz M, Thibaud E. Pubertal menorrhagia. J Pediatr Adolesc Gynaecol. 1996;9:16.

DOI: $10.5455 / 2320-1770 . i j \operatorname{cog} 20140941$

Cite this article as: Koranne PS, Wahane AR.

Puberty menorrhagia in modern era: analysis in a tertiary care centre. Int J Reprod Contracept Obstet Gynecol 2014;3:622-6. 